\title{
Quantum uncertainty relation saturated by the eigenstates of the harmonic oscillator
}

\author{
A. Mandilara and N. J. Cerf \\ Quantum Information and Communication, École Polytechnique de Bruxelles, CP 165/59, Université Libre de Bruxelles, \\ 1050 Brussels, Belgium
}

(Received 23 December 2011; published 24 September 2012)

\begin{abstract}
We rederive the Schrödinger-Robertson uncertainty principle for the position and momentum of a quantum particle. Our derivation does not directly employ commutation relations, but works by reduction to an eigenvalue problem related to the harmonic oscillator, which can then be further exploited to find a larger class of constrained uncertainty relations. We derive an uncertainty relation under the constraint of a fixed degree of Gaussianity and prove that, remarkably, it is saturated by all eigenstates of the harmonic oscillator. This goes beyond the common knowledge that the (Gaussian) ground state of the harmonic oscillator saturates the uncertainty relation.
\end{abstract}

DOI: 10.1103/PhysRevA.86.030102

PACS number(s): 03.65.-w, 03.67.-a, 42.50.-p

The Heisenberg uncertainty principle [1] captures the difference between classical and quantum states, and sets a limit on the precision of incompatible quantum measurements. It has been introduced in the early days of quantum mechanics, but its form has evolved with the understanding and formulation of quantum physics throughout the years. The first rigorous mathematical proof of Heisenberg's uncertainty relation for the canonical operators of position $\hat{x}$ and momentum $\hat{p}$ $([\hat{x}, \hat{p}]=i \hbar)$,

$$
\left(\left\langle\hat{x}^{2}\right\rangle-\langle\hat{x}\rangle^{2}\right)\left(\left\langle\hat{p}^{2}\right\rangle-\langle\hat{p}\rangle^{2}\right) \geqslant \hbar^{2} / 4,
$$

is due to Kennard [2] and Weyl [3], but only pure states were considered there. The full proof was later derived following different methodologies [4-6] (see Ref. [7] for more details), while the properties of the states saturating this inequality were also progressively unveiled.

The original uncertainty relation (1) only concerned the operators $\hat{x}$ and $\hat{p}$, but it was generalized to any pair of Hermitian operators by Schrödinger [8] and Robertson [9], in the case of pure states. In the same works, the anticommutator of $\hat{x}$ and $\hat{p}$ was also included in Eq. (1), yielding a stronger uncertainty relation,

$$
\begin{aligned}
& \left(\left\langle\hat{x}^{2}\right\rangle-\langle\hat{x}\rangle^{2}\right)\left(\left\langle\hat{p}^{2}\right\rangle-\langle\hat{p}\rangle^{2}\right) \\
& \quad-\frac{1}{4}(\langle\hat{x} \hat{p}+\hat{p} \hat{x}\rangle-2\langle\hat{x}\rangle\langle\hat{p}\rangle)^{2} \geqslant \hbar^{2} / 4,
\end{aligned}
$$

that bears their name. The first proof of the SchrödingerRobertson (SR) uncertainty relation for position and momentum, Eq. (2), in the general case including mixed states is probably due to Moyal [5], and, for any pair of not necessarily Hermitian operators, to Dodonov, Kurmyshev, and Man'ko [10]. In this latter work, the states of minimum uncertainty or minimizing states (MSs) for the SR inequality were identified as the pure states with a Gaussian wave function. Such Gaussian states are ubiquitous in physics as they play a major role, for example, in quantum optics (e.g., coherent states of the light field [11]), atomic physics (e.g., collective excitations of an atomic ensemble [12]), optomechanics (e.g., nanomechanical oscillators [13]), supraconductivity (e.g., superconducting LC circuits [14]), etc.

In this Rapid Communication, we revisit the status of Gaussian states in the context of uncertainty relations by exhibiting a connection with the harmonic oscillator and showing that, remarkably, all its eigenstates-not just its ground state-appear as minimum uncertainty states. We first rederive the SR inequality and corresponding MSs by using a variational method and standard algebraic tools. This derivation reveals the direct link between the quadratic order of Eqs. (1) and (2) in $\hat{x}$ and $\hat{p}$ and the fact that we deal with the quadratic Hamiltonian of a harmonic oscillator. Then, we move on to find bounded uncertainty relations [15], which give stronger bounds than Eq. (2) for states on which some a priori information is known, such as their purity [7] or entropy [16]. Specifically, we derive a Gaussianity-bounded uncertainty relation, depending on the degree of Gaussianity of the state as measured by a parameter $g$ that we introduce. We identify its corresponding set of MSs and find among them all the eigenstates of the harmonic oscillator. This yields a fundamental set of non-Gaussian minimum-uncertainty states, going beyond the common knowledge on the Heisenberg principle.

Although the uncertainty relations, being at the root of quantum mechanics, have been investigated in various situations, such as multidimensional $[17,18]$ or mixed states $[7,16,19]$, our results imply that there is more to gain by analyzing them under the perspective of the Gaussian character of a state. Non-Gaussian states of light can now be handled in the laboratory [20-25] and have been proven essential in the field of continuous-variable quantum information [26-30], but they remain hard to classify. Identifying states of minimum uncertainty among them may lead to a better understanding of the structure of the state space in infinite dimension and, since the Heisenberg principle is at the heart of the limitations on measurement precision [31], to further developments in quantum metrology.

Unconstrained SR relation. We introduce our method as a way to find the MS of the SR uncertainty relation. Consider a quantum state characterized by its density operator $\hat{\rho}$. Its covariance matrix is defined as

$$
\gamma=\left(\begin{array}{cc}
\left\langle\hat{x}^{2}\right\rangle-\langle\hat{x}\rangle^{2} & \frac{1}{2}\langle\hat{x} \hat{p}+\hat{p} \hat{x}\rangle-\langle\hat{x}\rangle\langle\hat{p}\rangle \\
\frac{1}{2}\langle\hat{x} \hat{p}+\hat{p} \hat{x}\rangle-\langle\hat{x}\rangle\langle\hat{p}\rangle & \left\langle\hat{p}^{2}\right\rangle-\langle\hat{p}\rangle^{2}
\end{array}\right),
$$

where $\langle\cdot\rangle=\operatorname{Tr}(\hat{\rho} \cdot)$ stand for quantum expectation values in state $\hat{\rho}$. Hereafter, we define the uncertainty of the state $\hat{\rho}$ as the dimensionless variable

$$
\alpha=2(\operatorname{det} \gamma)^{1 / 2} / \hbar
$$


which is simply the square root of the left-hand side of Eq. (2) divided by $\hbar / 2$. We write it with the determinant of $\gamma$ to emphasize that it remains invariant under any linear canonical transformation in $\hat{x}$ and $\hat{p}$, that is, under any operation modeled by a Hamiltonian that is quadratic in $\hat{x}$ and $\hat{p}$ (see the Supplemental Material [32]). This brings the strong simplification that it is sufficient to confine our search for MSs among states that satisfy the constraints

$$
\begin{gathered}
\operatorname{Tr}(\hat{\rho} \hat{x})=\operatorname{Tr}(\hat{\rho} \hat{p})=0, \\
\operatorname{Tr}(\hat{\rho}(\hat{x} \hat{p}+\hat{p} \hat{x}))=\operatorname{Tr}\left(\hat{\rho}\left(\hat{x}^{2}-\hat{p}^{2}\right)\right)=0,
\end{gathered}
$$

that is, whose mean values vanish and $\gamma$ is proportional to the identity. Under these conditions, Eq. (4) can be expressed as

$$
\alpha=\operatorname{Tr}[\hat{\rho}(1+2 \hat{n})],
$$

where $\hat{n}=\left(\hat{x}^{2}+\hat{p}^{2}-1\right) / 2$ is the number operator for the harmonic oscillator (from now on, we assume $\hbar=1$ and take the particle's mass and angular frequency equal to one).

Now, let us proceed with the minimization of $\alpha$ under constraints (5) and (6) by using the Lagrange multipliers method. For any density operator $\hat{\rho}$, there is an eigenbasis $\left\{\left|\Psi_{n}\right\rangle\right\}$ such that $\hat{\rho}=\sum c_{n}\left|\Psi_{n}\right\rangle\left\langle\Psi_{n}\right|$ with $0 \leqslant c_{n} \leqslant 1$ and $\sum c_{n}=1$. It is more convenient to define the unnormalized vectors $\left|\psi_{n}\right\rangle=\sqrt{c_{n}}\left|\Psi_{n}\right\rangle$ and rewrite the state as $\hat{\rho}=\sum_{n}\left|\psi_{n}\right\rangle\left\langle\psi_{n}\right|$, while imposing the additional constraint

$$
\operatorname{Tr}(\hat{\rho})=1 \text {. }
$$

Then, choosing an orthonormal basis $\{|i\rangle\}$ to decompose the vectors $\left|\psi_{n}\right\rangle=\sum \psi_{n}^{i}|i\rangle$, we can reexpress the uncertainty (7) and constraints (5), (6), and (8) as functions of the $\psi_{n}^{i}$, s. We define the Lagrange multipliers $\lambda_{k}^{\prime}$ and consider the "uncertainty" functional

$$
\begin{aligned}
\tilde{\alpha}= & \alpha+\lambda_{1}^{\prime} \operatorname{Tr}(\hat{\rho})+\lambda_{2}^{\prime} \operatorname{Tr}(\hat{\rho} \hat{x})+\lambda_{3}^{\prime} \operatorname{Tr}(\hat{\rho} \hat{p}) \\
& +\lambda_{4}^{\prime} \operatorname{Tr}[\hat{\rho}(\hat{x} \hat{p}+\hat{p} \hat{x})]+\lambda_{5}^{\prime} \operatorname{Tr}\left[\hat{\rho}\left(\hat{x}^{2}-\hat{p}^{2}\right)\right],
\end{aligned}
$$

which implicitly depends on the complex amplitudes $\psi_{n}^{i}$,s. Extremizing $\tilde{\alpha}$ yields conditions on these amplitudes (see the Supplemental Material [32]), which read as conditions on the unnormalized eigenvectors $\left|\psi_{n}\right\rangle$ defining the minimizing state $\hat{\rho}$, namely,

$$
\begin{aligned}
& {\left[\hat{n}+1 / 2+\lambda_{1}+\lambda_{2} \hat{x}+\lambda_{3} \hat{p}+\lambda_{4}(\hat{x} \hat{p}+\hat{p} \hat{x})\right.} \\
& \left.\quad+\lambda_{5}\left(\hat{x}^{2}-\hat{p}^{2}\right)\right]\left|\psi_{n}\right\rangle=0,
\end{aligned}
$$

where $\lambda_{k}=2 \lambda_{k}^{\prime}$. Introducing the Hermitian operator

$$
\hat{H}=\hat{n}+\frac{1}{2}+\lambda_{2} \hat{x}+\lambda_{3} \hat{p}+\lambda_{4}(\hat{x} \hat{p}+\hat{p} \hat{x})+\lambda_{5}\left(\hat{x}^{2}-\hat{p}^{2}\right),
$$

we can rewrite Eq. (10) as $\hat{H}\left|\psi_{n}\right\rangle=-\lambda_{1}\left|\psi_{n}\right\rangle, \forall n$, leading to the necessary condition that the eigenvectors $\left|\psi_{n}\right\rangle$ defining the MS must be degenerate eigenvectors of $\hat{H}$ corresponding all to the same eigenvalue.

Thus, one should diagonalize $\hat{H}$ in order to proceed with the identification of the MS. As explained in the Supplemental Material [32], there exists a linear canonical transformation in $x$ and $p$ that transforms $\hat{H}$ onto the Hamiltonian of the harmonic oscillator, $\hat{H}_{0}=\hat{n}+1 / 2$. Obviously, this means that $\hat{H}$ has the same eigenvalues as $\hat{H}_{0}$ and that its eigenvectors are $U|n\rangle$, where $|n\rangle$ are the number states (eigenstates of $\hat{H}_{0}$ ) and $U$ is the Gaussian unitary corresponding to this canonical transformation. Since $\hat{H}_{0}$ does not possess any degeneracy in its spectrum, the same holds for $\hat{H}$ and therefore the only possibility is that the MS is a pure state of the type $\hat{\rho}=U|n\rangle\langle n| U^{\dagger}$. Among these states, we must keep those satisfying constraints (5) and (6), which are the number states $|n\rangle$, so that the state that minimizes the uncertainty (7) is obviously the ground state $|0\rangle$. Of course, by plugging $\hat{\rho}=|0\rangle\langle 0|$ into Eq. (7), we recover the lower bound of the SR relation, $\hbar^{2} / 4$. By acting with linear canonical transformations on $|0\rangle$, we obtain all Gaussian pure states, which is the wellknown set of MSs for the SR uncertainty relation [32]. This was a long detour to rederive Eq. (2), but this connection with the harmonic oscillator turns out to be crucial in what follows.

Degree of Gaussianity. Our method works by reduction to a constrained optimization problem (even for solving the unconstrained SR inequality), so it can be simply adapted to find the MS with an extra constraint on Gaussianity. Several measures of non-Gaussianity have been used in the literature [15,16,33-35], but we instead suggest using a parameter $g$ capturing the degree of Gaussianity, inspired from our former work on non-Gaussian states with a positive Wigner function [36,37]. Denoting as $\hat{\rho}_{G}$ the Gaussian state that has the same covariance matrix $\gamma$ (and same mean values $\langle\hat{x}\rangle$ and $\langle\hat{p}\rangle$ ) as state $\hat{\rho}$, we define the Gaussianity of $\hat{\rho}$ as

$$
g=\operatorname{Tr}\left(\hat{\rho} \hat{\rho}_{G}\right) / \operatorname{Tr}\left(\hat{\rho}_{G}^{2}\right) .
$$

It is more appropriate for our purposes and also has merits on its own. Its main properties are as follows (see the Supplemental Material [32] for the proofs): (i) $g$ is invariant under linear canonical transformations in $x$ and $p$; (ii) $g$ is a bounded quantity, that is, $2 / e \leqslant g \leqslant 2$, and $g=1$ for Gaussian states (but the converse is not true); and (iii) $g$ provides a necessary criterion for the strict positivity of the Wigner function of a state.

Let us briefly address possible experimental means for a direct estimation of $g$ without going through a full state tomography procedure of $\hat{\rho}$ (see the Supplemental Material [32]). The trace overlap $\operatorname{Tr}\left(\hat{\rho} \hat{\rho}_{G}\right)$ can be estimated by using the eight-port homodyne detection scheme that is usually employed to estimate the $Q$ function of a state in quantum optics by simultaneously measuring the $x$ and $p$ quadratures of the two output modes of a balanced beam splitter [11]. If we inject the state $\hat{\rho}$ together with $\hat{\rho}_{G}$ (instead of the vacuum state $|0\rangle$ ) in this beam splitter preceding the quadrature measurements, then the value of the modified $Q$ function at the origin would read $Q(0,0)=\operatorname{Tr}\left(\hat{\rho} \hat{\rho}_{G}\right)$. This method for measuring $g$ would, however, require first performing homodyne measurements on $\hat{\rho}$ in order to obtain its covariance matrix $\gamma$ and prepare the Gaussian state $\hat{\rho}_{G}$.

Gaussianity-bounded SR relation. Our optimization method provides a necessary condition on the extremal solutions since it relies on the Lagrange multipliers method, so that concluding on a solution may become complicated if the eigenvectors of $\hat{H}$ cannot be identified analytically. Fortunately, finding an uncertainty relation under a fixed- $g$ constraint leads to an eigenvalue problem that is analytically solvable. As before, we can confine our search on states satisfying constraints (5) and (6) since neither $\alpha$ nor $g$ change under linear canonical transformations. For these states, the 
corresponding Gaussian state $\hat{\rho}_{G}$ can be expressed simply as

$$
\hat{\rho}_{G}=e^{-\beta \hat{n}} / N
$$

where $e^{-\beta}=\frac{\alpha-1}{\alpha+1}$ and $N=(\alpha+1) / 2$. In addition, $\operatorname{Tr}\left(\hat{\rho}_{G}^{2}\right)=$ $1 / \alpha$. Instead of minimizing the uncertainty $\alpha$ for a fixed Gaussianity $g$, it is easier to fix $\alpha$ and search for states of extremal $g$, or extremal overlap $o=\operatorname{Tr}\left(\hat{\rho} e^{-\beta \hat{n}}\right)$. The variational procedure for deriving the MS is completely analogous, but we extremize $o$ using a constraint on the uncertainty (7) in addition to Eqs. (5), (6), and (8). Thus, the "overlap" functional is

$$
\begin{aligned}
\tilde{o}= & o+\lambda_{1}^{\prime} \operatorname{Tr}(\hat{\rho})+\lambda_{2}^{\prime} \operatorname{Tr}(\hat{\rho} \hat{x})+\lambda_{3}^{\prime} \operatorname{Tr}(\hat{\rho} \hat{p}) \\
& +\lambda_{4}^{\prime} \operatorname{Tr}[\hat{\rho}(\hat{x} \hat{p}+\hat{p} \hat{x})]+\lambda_{5}^{\prime} \operatorname{Tr}\left[\hat{\rho}\left(\hat{x}^{2}-\hat{p}^{2}\right)\right] \\
& +\lambda_{6}^{\prime} \operatorname{Tr}[\hat{\rho}(2 \hat{n}+1)],
\end{aligned}
$$

depending on six Lagrange multipliers $\lambda_{k}^{\prime}$. The extremization conditions on the unnormalized eigenvectors $\left|\psi_{n}\right\rangle$ of the solution state can be written as

$$
\begin{aligned}
& {\left[e^{-\beta \hat{n}}+1 / 2+\lambda_{1}+\lambda_{2} \hat{x}+\lambda_{3} \hat{p}+\lambda_{4}(\hat{x} \hat{p}+\hat{p} \hat{x})\right.} \\
& \left.+\lambda_{5}\left(\hat{x}^{2}-\hat{p}^{2}\right)+\lambda_{6} \hat{n}\right]\left|\psi_{n}\right\rangle=0 .
\end{aligned}
$$

where $\lambda_{k}=2 \lambda_{k}^{\prime}$. By defining the Hermitian operator

$$
\begin{aligned}
\hat{H}_{1}= & e^{-\beta \hat{n}}+\lambda_{2} \hat{x}+\lambda_{3} \hat{p}+\lambda_{4}(\hat{x} \hat{p}+\hat{p} \hat{x}) \\
& +\lambda_{5}\left(\hat{x}^{2}-\hat{p}^{2}\right)+\lambda_{6} \hat{n},
\end{aligned}
$$

we conclude that $\left|\psi_{n}\right\rangle$ should be degenerate eigenvectors of $\hat{H}_{1}$. It can be shown that, without loss of generality, we can restrict ourselves to states $\left|\psi_{n}\right\rangle=\psi_{n}|n\rangle$, with $\psi_{n}$ being complex amplitudes [32]. For these states, the constraints (5) and (6) are satisfied, and $\hat{H}_{1}$ is replaced by

$$
\hat{H}_{2}=e^{-\beta \hat{n}}+\lambda_{6} \hat{n} \text {. }
$$

The eigenvectors of $\hat{H}_{2}$ are the number states $|n\rangle$, but, unlike for the harmonic oscillator, double degeneracies are possible if $\lambda_{6}<0$. As a result, we look for mixtures of two number states $\hat{\rho}=\left|\psi_{n}\right|^{2}|n\rangle\left\langle\left. n|+| \psi_{m}\right|^{2} \mid m\right\rangle\langle m|$ satisfying the normalization constraint $\left|\psi_{n}\right|^{2}+\left|\psi_{m}\right|^{2}=1$ and uncertainty constraint

$$
\left|\psi_{n}\right|^{2}(2 n+1)+\left|\psi_{m}\right|^{2}(2 m+1)=\alpha
$$

that achieve the minimum or maximum

$$
g=\left|\psi_{n}\right|^{2} \frac{2 \alpha(\alpha-1)^{n}}{(\alpha+1)^{n+1}}+\left|\psi_{m}\right|^{2} \frac{2 \alpha(\alpha-1)^{m}}{(\alpha+1)^{m+1}} .
$$

By supervision, one can see that the minimum $g$ (corresponding to positive eigenvalues of $\hat{H}_{2}$ ) is achieved by mixtures of two successive number states

$$
\hat{\rho}_{\text {min }}=r|n\rangle\langle n|+(1-r)| n+1\rangle\langle n+1|,
$$

with the parameters $n$ and $r \in[0,1$ [ depending on $\alpha$. The number states $|n\rangle$ are naturally included in the set for $r=0$. The maximum $g$ (corresponding to negative eigenvalues of $\left.\hat{H}_{2}\right)$ is achieved by mixtures

$$
\hat{\rho}_{\max }=r|0\rangle\langle 0|+(1-r)| n\rangle\langle n|,
$$

in the limit $n \rightarrow \infty, r \rightarrow 1$, while $\alpha=r+(1-r)(2 n+1)$ is kept finite.

In Fig. 1, we plot as a dashed line the two extremal values of $g$ for a fixed $\alpha$ as realized by the states of Eqs. (20) and (21). The MSs (i.e., the states minimizing the uncertainty $\alpha$ for a fixed degree of Gaussianity $g$ ) correspond only to some
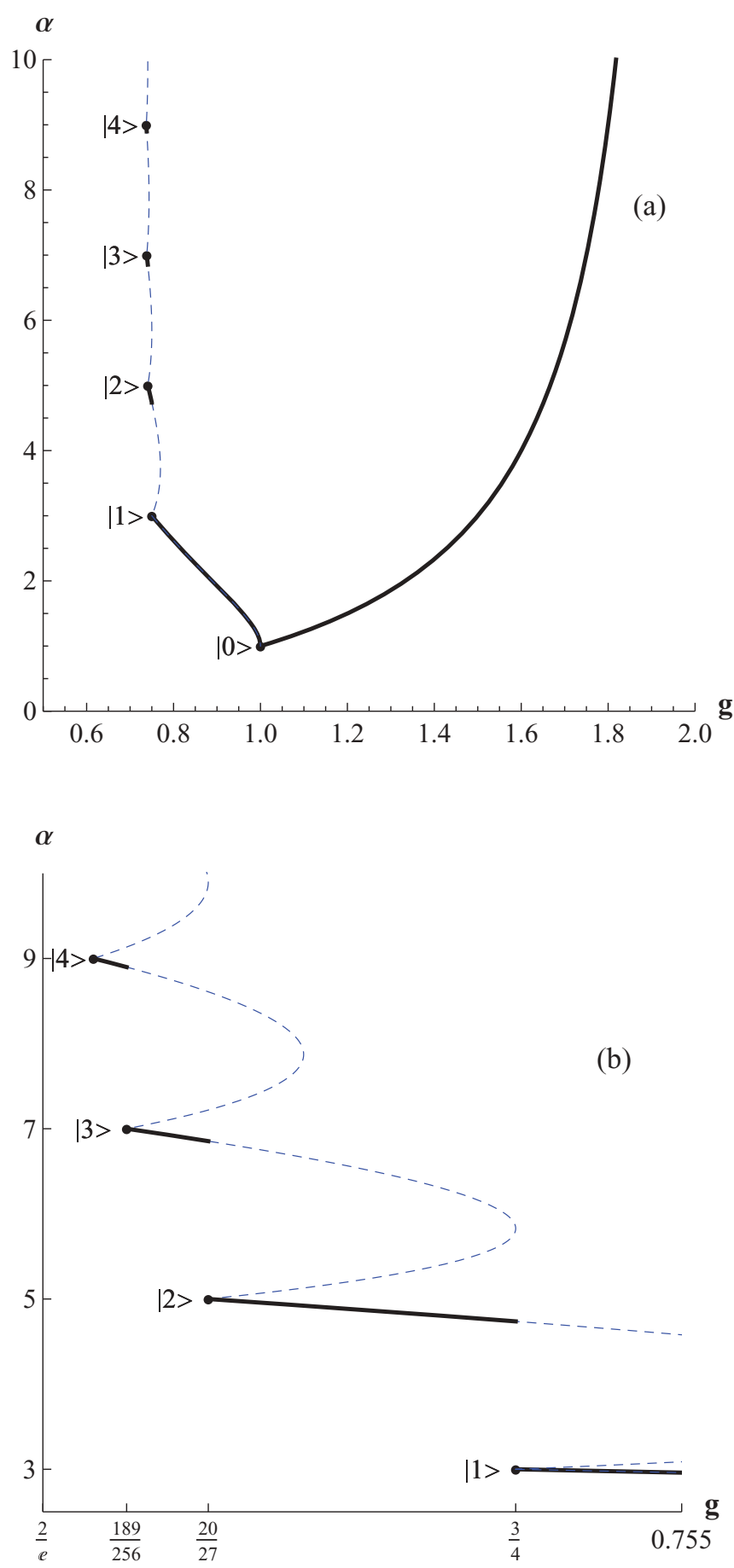

FIG. 1. (Color online) (a) Extremal values of the degree of Gaussianity $g$ for a fixed uncertainty $\alpha$ shown as a dashed (blue) line. It is achieved by states $\hat{\rho}_{\min }$ for $g \leqslant 1$, and $\hat{\rho}_{\max }$ for $g>1$. The line connecting subsequent number states $|n\rangle$ and $|n+1\rangle$ is realized by mixtures of them. The Gaussianity-bounded SR relation corresponds to the part of this extremal line shown as a solid (black) line. The uncertainty $\alpha$ must have a value larger or equal to the solid line for a given $g$. (b) Magnified view of (a), where the discontinuity of the uncertainty relation becomes evident.

part of this line, which we show as a solid line. For $g>1$, the situation is simple and all states $\hat{\rho}_{\max }$ are MSs. For this region, the minimum uncertainty as a function of $g$ can be 
easily derived as

$$
\alpha \geqslant \frac{g}{2-g},
$$

employing Eq. (21) (see [32]). In contrast, for $\frac{2}{e}<g \leqslant 1$, the minimum $\alpha$ for fixed $g$ displays discontinuities. In the interval

$$
\frac{(n+1)^{n+1}(3+2 n)}{(2+n)^{2+n}}<g \leqslant \frac{n^{n}(1+2 n)}{(1+n)^{1+n}},
$$

$\alpha$ is minimized by the states $\hat{\rho}_{\text {min }}$ with the value of $n$ satisfying Eq. (23). In particular, we see that all number states $|n\rangle$ are included in this set for specific values of $g$ corresponding to the upper bound of Eq. (23) for different $n$ 's. Moreover, in Fig. 1(b), we see that most of the MSs (solid line) consist of these states $|n\rangle$ and their close neighborhood. Thus, as advertised, we conclude that all eigenstates of the harmonic oscillator are extremal in the sense that they exhibit the lowest allowed uncertainty given their non-Gaussian character.

For other values of $g$ in the interval $\frac{2}{e}<g \leqslant 1$, once the value of $n$ is identified from Eq. (23), one has to solve the polynomial equation

$$
4 \alpha \frac{(\alpha-1)^{n}}{(\alpha+1)^{2+n}}(1+n)=g
$$

for $\alpha$ in order to find the dependence of the uncertainty $\alpha$ on the Gaussianity $g$. For $3 / 4<g \leqslant 1$, which covers most of the interesting region, the explicit expression [32] is

$$
\alpha \geqslant \frac{2+2 \sqrt{1-g}-g}{g} .
$$

In the Supplemental Material [32] we also show that in addition to the states $\hat{\rho}_{\min }$ and $\hat{\rho}_{\max }$, which are phase invariant, the set of MSs comprises all states with a covariance matrix proportional to the identity which can be transformed onto them by phase averaging. Furthermore, all states connected to $\hat{\rho}_{\min }$ and $\hat{\rho}_{\max }$ by linear canonical transformations are MSs as well, since the uncertainty $\alpha$ and Gaussianity $g$ are invariants of the group.
Conclusions. We have exhibited a variational method to derive the Schrödinger-Robertson uncertainty relation by casting it as an eigenvalue problem related to the harmonic oscillator. It follows an "inverse path" to the common procedure where the lower bound on the uncertainty is derived based on commutators, and the MSs are then identified. Such an inverse procedure was put forward by Dodonov and Man'ko for the derivation of purity-bounded uncertainty relations [7,15], but it appears that our method is more generally applicable because it is based on the amplitudes of the eigenstates of the MSs instead of its density matrix elements [32]. It is especially useful when constraints are included that account for some knowledge on the state.

In particular, we have found an uncertainty relation that is bounded by the degree of Gaussianity $g$ of the state. The state with the overall lowest uncertainty $\alpha$ is of course the Gaussian ground state $|0\rangle$ of the harmonic oscillator $(g=1)$, but we have proven that the non-Gaussian states with the lowest uncertainty $\alpha$ for a fixed $g<1$ include as well mixtures of subsequent number states $|n\rangle$ of the harmonic oscillator. Among these $\mathrm{MSs}$, the number states play a prominent role as they are the only phase-invariant pure states. We have thus proven that the number states are also extremal in this uncertainty-related sense, thereby extending to all (non-Gaussian) eigenstates of the harmonic oscillator the celebrated minimum-uncertainty property of its (Gaussian) ground state. Given the considerable attention that non-Gaussian states are attracting in continuousvariable quantum information theory (see, e.g., Ref. [38]), unveiling this extremality property of harmonic oscillator states may contribute to further fundamental progress in the field, especially in relation to quantum metrology.

A.M. gratefully acknowledges financial support from the F.R.S-FNRS. This work was also carried out with the financial support of the F.R.S-FNRS via project HIPERCOM and the support of the Belgian Federal program IUAP via project Photonics@be.
[1] W. Heisenberg, Z. Phys. 43, 172 (1927).

[2] E. H. Kennard, Z. Phys. 44, 326 (1927).

[3] H. Weyl, Theory of Groups and Quantum Mechanics (Dutton, New York, 1927), pp. 77, 393, 394.

[4] L. I. Mandel'shtam and I. E. Tamm, Izv. AN USSR, Ser. Fiz. 9, 122 (1945).

[5] J. E. Moyal, Proc. Cambridge Philos. Soc. 45, 99 (1949).

[6] D. Stoler and S. Newman, Phys. Lett. A 38, 433 (1972).

[7] V. V. Dodonov and V. I. Man'ko, in Invariants and Evolution of Nonstationary Quantum Systems (Proceedings of the Lebedev Physics Institute), edited by M. A. Markov (Nova Science, Commack, NY, 1989), Vol. 183, pp. 3-101.

[8] E. Schrödinger, Sitzungsber. Preuss. Akad. Wiss. 14, 296 (1930).

[9] H. P. Robertson, Phys. Rev. 35, 667A (1930); 46, 794 (1934).

[10] V. V. Dodonov, E. V. Kurmyshev, and V. I. Man'ko, Phys. Lett. A 79, 150 (1980).
[11] M. O. Scully and M. S. Zubairy, Quantum Optics (Cambridge University Press, Cambridge, UK, 1997).

[12] K. Hammerer, A. Sorensen, and E. S. Polzik, Rev. Mod. Phys. 82, 1041 (2010).

[13] S. Groeblacher, K. Hammerer, M. R. Vanner, and M. Aspelmeyer, Nature (London) 460, 724 (2009).

[14] I. Chiorescu, Y. Nakamura, C. J. P. M. Harmans, and J. E. Mooij, Science 299, 1869 (2003).

[15] V. V. Dodonov, J. Opt. B 4, S98 (2002).

[16] M. J. Bastiaans, J. Opt. Soc. Am. A 3, 1243 (1986).

[17] E. C. G. Sudarshan, C. B. Chiu, and G. Bhamathi, Phys. Rev. A 52, 43 (1995).

[18] R. Simon, N. Mukunda, and B. Dutta, Phys. Rev. A 49, 1567 (1994).

[19] S. A. Ponomarenko and E. Wolf, Phys. Rev. A 63, 062106 (2001); G. S. Agarwal and S. A. Ponomarenko, ibid. 67, 032103 (2003). 
[20] J. S. Neergaard-Nielsen, B. M. Nielsen, C. Hettich, K. Molmer, and E. S. Polzik, Phys. Rev. Lett. 97, 083604 (2006).

[21] A. Ourjoumtsev, H. Jeong, R. Tualle-Brouri, and P. Grangier, Nature (London) 448, 784 (2007).

[22] V. Parigi, A. Zavatta, M. Kim, and M. Bellini, Science 317, 1890 (2007).

[23] H. Takahashi, K. Wakui, S. Suzuki, M. Takeoka, K. Hayasaka, A. Furusawa, and M. Sasaki, Phys. Rev. Lett. 101, 233605 (2008).

[24] A. Zavatta, V. Parigi, M. S. Kim, H. Jeong, and M. Bellini, Phys. Rev. Lett. 103, 140406 (2009).

[25] E. Bimbard, N. Jain, A. MacRae, and A. I. Lvovsky, Nat. Photonics 4, 243 (2010).

[26] J. Eisert, S. Scheel, and M. B. Plenio, Phys. Rev. Lett. 89, 137903 (2002).

[27] J. Fiurasek, Phys. Rev. Lett. 89, 137904 (2002).

[28] G. Giedke and J. I. Cirac, Phys. Rev. A 66, 032316 (2002).

[29] J. Niset, J. Fiurasek, and N. J. Cerf, Phys. Rev. Lett. 102, 120501 (2009).

[30] L. Magnin, F. Magniez, A. Leverrier, and N. J. Cerf, Phys. Rev. A 81, 010302(R) (2010).
[31] S. L. Braunstein, C. M. Caves, and G. J. Milburn, Ann. Phys. 247, 135 (1996).

[32] See Supplemental Material at http://link.aps.org/supplemental/ 10.1103/PhysRevA.86.030102 for details on the proofs, methods, and the definition of the degree of Gaussianity.

[33] M. G. Genoni, M. G. A. Paris, and K. Banaszek, Phys. Rev. A 76, 042327 (2007).

[34] M. G. Genoni, M. G. A. Paris, and K. Banaszek, Phys. Rev. A 78, 060303(R) (2008); M. G. Genoni and M. G. A. Paris, ibid. 82, 052341 (2010).

[35] J. S. Ivan, M. S. Kumar, and R. Simon, Quantum Inf. Process. 11, 853 (2012).

[36] A. Mandilara, E. Karpov, and N. J. Cerf, Phys. Rev. A 79, 062302 (2009).

[37] A. Mandilara, E. Karpov, and N. J. Cerf, J. Phys.: Conf. Ser. 254, 012011 (2010).

[38] Quantum Information with Continuous Variables of Atoms and Light, edited by N. J. Cerf, G. Leuchs, and E. S. Polzik (Imperial College Press, London, 2007). 